\title{
Development of Rofecoxib-Based Fluorescent Probes and Investigations on Their AIE Activity, Solvatochromism, Mechanochromism and COX-2 Targeted Bioimaging
}

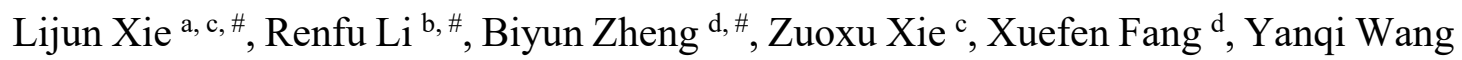

${ }^{\mathrm{b}}$, Gregory Cuny ${ }^{\mathrm{c}}$, Zhenli Li ${ }^{\mathrm{e}}$, Bin Lin ${ }^{\mathrm{e},}{ }^{*}$, Xueyuan Chen ${ }^{\mathrm{b}, *}$, Ming Hu ${ }^{\mathrm{c}, *}$

${ }^{a}$ Fujian Provincial Key Laboratory of Screening for Novel Microbial Products, Fujian Institute of Microbiology, Fuzhou, Fujian 350007, PR China

${ }^{\mathrm{b}}$ CAS Key Laboratory of Design and Assembly of Functional Nanostructures, and Fujian Key Laboratory of Nanomaterials, Fujian Institute of Research on the Structure of Matter, Chinese Academy of Sciences, Fuzhou, Fujian 350002, PR China

${ }^{\mathrm{c}}$ Department of Pharmacological and Pharmaceutical Sciences, College of Pharmacy, University of Houston, Houston, TX 77204

${ }^{\mathrm{d}}$ Department of Gastroenterology, Fujian Medical University Union Hospital, Fuzhou, Fujian 350007, PR China 
e Department of Medicinal Chemistry, School of Pharmaceutical Engineering, Shenyang Pharmaceutical University, Shenyang, Liaoning 110016, PR China

ABSTRACT: Cyclooxygenase-2 (COX-2) fluorescent probes are promising tools for early cancer diagnosis. Traditionally, COX-2 probes were designed by connecting two parts, a fluorophore and a COX-2 binding unit, via a flexible linker. Herein, a new class of COX-2-specific fluorescent probes have been developed by one-step modification from rofecoxib by an integrative approach to combining the fluorophore and binding units into one. Among them, several new rofecoxib analogues not only retained their COX-2 binding ability but also exhibited attractive fluorescent properties, such as tunable Blue-Red emission, solvatochromism, AIE behavior and mechanochromism. Notably, the emission of $\mathbf{2 a 1 6}$ can be switched between greenish-yellow in the crystalline state to red-orange in the amorphous state by grinding and fuming treatments. Furthermore, the highly fluorescent compound $\mathbf{2 a 1 6}\left(\Phi_{\mathrm{f}}=0.94\right.$ in powder $)$ displayed much stronger fluorescent imaging of COX-2 in HeLa cancer cells overexpressing COX-2 than RAW264.7 normal cells with minimal expressing of COX-2. Most importantly, 2a16 can light up human cancer tissues from adjacent normal tissues with much brighter fluorescence by targeting COX-2 enzyme. These results illustrate the potential of $2 \mathbf{a 1 6}$ as a new red fluorescent probe for human cancer imaging in clinical applications. 
KEYWORDS: COX-2 Fluorescent probes, Cancer imaging, AIEgens,

Mechanochromism, Rofecoxib. 


\section{Introduction}

Cancer has become one of the major causes of death worldwide and death can be prevented in $30 \%$ of the cancer patients if they are diagnosed and treated during the early stages of cancer progression. ${ }^{[1-2]}$ Therefore, an early detection strategy is critical to reduce mortality from cancer. In the past two decades, molecular fluorescence microscopy imaging has come to offer opportunities for early detection of cancer due to its advantages such as high selectivity, high resolution, and noninvasive capabilities. It is highly dependent on the fluorescent capability of the molecular probes whose fluorescent signals are generated by enzymic biomarkers in the cancer cells or tissues. Therefore, enormous efforts have been devoted to developing efficient and convenient molecular probes for the detection of all kinds of cancers.

Accumulating research data have demonstrated that cyclooxygenase- 2 (COX-2) is an attractive biomarker for early detection and diagnosis of cancer, because it is overexpressed at all stages of cancer, but generally not or poorly expressed in normal tissues. ${ }^{[3-10]}$ Such different COX-2 levels make it possible to detect cancer at an early stage if a suitable COX-2 fluorescent probe is used.

In recent years, a large number of COX-2 fluorescent probes have been developed and they were shown to have the ability to detect the expression level of COX-2 in cells and cancer tissues. However, to the best of our knowledge, all the COX-2 fluorescent probes developed so far are based on the strategy by which a fluorophore is coupled to a binding unit via a flexible linker. ${ }^{[9-20]}$ The binding groups used so far are celecoxib, indomathacin, and indomathacin derivatives. Previously, by following this strategy, we 
developed a COX-2 fluorescent probe CCY-5 by linking CY-5 as the fluorophore to celecoxib as the binding unit (Figure 1A). ${ }^{[21]}$ In the process of designing CCY -5 , we investigated various COX-2 inhibitors with diverse scaffolds, such as rofecoxib, celecoxib, indomethacin, (S)-naproxen and acetyl salicylic acid. Among them, celecoxib, indomethacin, and rofecoxib are commonly applied because they are potent COX inhibitors. ${ }^{[22-23]}$ Of these three, rofecoxib attracted our attention because of the existence of half tetraphenylethene (TPE) scaffold in its structure, which is well known as a classic aggregation induced emission (AIE) material. ${ }^{[24-25]}$ Meanwhile, the electron withdrawing group (EWG), methylsulfonyl on the $\mathrm{Ar}^{2}$ ring makes it possible to construct a new AIEgens by adding an electron donating group (EDG) to the other side of rofecoxib, resulting in the formation of D- $\pi$-A system (Figure 1B). ${ }^{[26-28]}$

Base on this strategy, we developed a new type of AIEgens by modifying rofecoxib with the extension of $\pi$-conjugation (Figure $1 \mathrm{~B}) .{ }^{[29-30]}$ In addition, the COX-2 binding ability of these compounds to the COX-2 enzyme was not affected because the binding moiety of rofecoxib's structure was kept intact. In the end, the fluorophore and binding moiety was merged into each other in this work instead of two separate parts connected via a linker as those in the literature. Such a strategy for developing novel COX-2 fluorescent probes offers some advantages over the traditional strategy, such as facile syntheses, tunable fluorescent properties and probes with smaller molecular weights.

Herein, we describe a facile one-step approach for modifying rofecoxib to develop novel AIEgens with COX-2 binding ability. $\mathrm{An}^{\mathrm{Ar}^{3}}$ ring is introduced to rofecoxib to endow its analogues with efficient solid-state AIE property (Figure 1B). Moreover, 
these AIEgens were found to possess red emission and large Stokes shifts, which enlarges their potential for COX-2 related biological imaging. ${ }^{[31-32]}$ Among the rofecoxib analogues, 2a16 with excellent fluorescent property $\left(\Phi_{\mathrm{f}}=0.94, \lambda \mathrm{em} / \mathrm{max}=\right.$ $677 \mathrm{~nm}$ ) showed the ability to distinguish cancer cells from normal cells. It was further evaluated in human cancer tissues by lighting up cancer tissues but not the adjacent normal tissues. $\mathbf{2 a 1 6}$ may be developed as a diagnostic reagent for diseases associated with increased COX-2 expression (e.g., cancer and colitis).

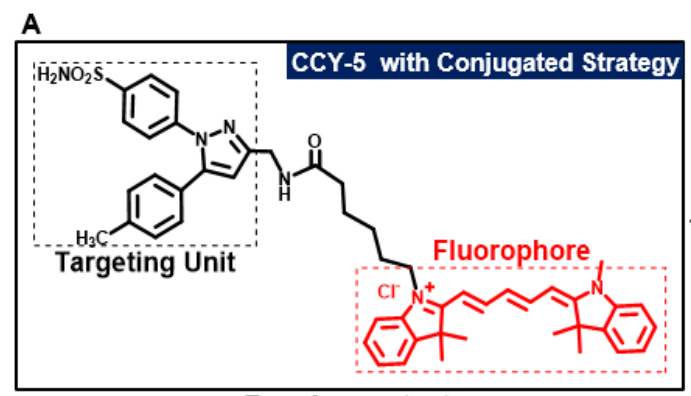

Previous strategy

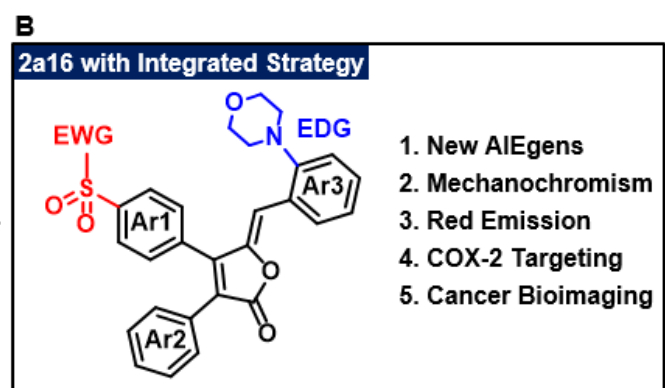

This strategy

Figure 1. (A) Previously reported CCY-5: celecoxib was conjugated with fluorophore CY-5 via a linker; (B) A new AIEgen fluorophore was developed by minor modification based on the structure of rofecoxib.

\section{Experimental Section}

Materials. All commercial reagents and solvents were used as received. Various benzaldehydes with different substituted group were purchased from Alfa Aesar (US). 4-methoxyphenylacetic acid, 2-bromo-p-methylsulfonyl acetophenone, were purchased from TCI (US). Rofecoxib was purchased from Yebang Chemicals, Inc. (China). Reactions were magnetically stirred and monitored on TLC Silica gel 60G F254 plate from Millipore Sigma. Flash column chromatography was performed using silica gel (40-63 um (230-400 mesh), $60 \AA$ irregular pore diameter). Dulbecco’s 
Modified Eagle Medium (DMEM) was purchased from Invitrogen (Carlsbad, CA). Fetal bovine serum (FBS) was purchased from Gibco, Life Technologies (Gaithersburg, MD). All other reagents and solvents were purchased from Sigma-Aldrich (US) at the highest commercial quality and used without further purification, unless otherwise stated.

Instruments. High-resolution mass spectra were obtained at the Mass Spectrometry Service Center of Fujian institute of microbiology on an Agilent 6545 Q-TOF LCMS. Nuclear magnetic resonance (NMR) spectra were obtained on a Bruker UNI-500 and AVII-500B instrument and JEOL 600MHz spectrometers (Framingham, MA, USA) using deuterated solvent (DMSO-d6). Photoluminescence (PL) excitation and emission spectra and PL decays were recorded on the FLS980 spectrometer (Edinburgh) equipped with both continuous Xenon $(450 \mathrm{~W})$. PL, AIE were taken by using a Canon 70D digital camera without using any filter. A differential scanning calorimetry (DSC) trace was obtained on a DSC Q2000 under nitrogen atmosphere. Powder X-ray diffraction (PXRD) patterns were recorded on a Rigaku (Ultima IV) diffractometer. Single crystal X-ray diffraction (SXRD) data were measured on a Bruker Smart APEX CCD-based diffractometer. The absolute PLQYs of sample were measured by employing a standard barium sulfate coated integrating sphere $(150 \mathrm{~mm}$ in diameter, Edinburgh) as the sample chamber that was mounted on the FLS980 spectrometer with the entry and output port of the sphere located in $90^{\circ}$ geometry from each other in the plane of the spectrometer. A standard tungsten lamp was used to correct the optical response of the instrument. All the spectral data were recorded at room temperature 
unless otherwise noted, and corrected for the spectral response of both the spectrometer and the integrating sphere.

Design and Syntheses of novel rofecoxib analogues. The designed rofecoxib analogues, which contain a five-membered lactone ring with an aromatic substituent in position 5 , were prepared by adding $0.12 \mathrm{~g}(0.0014 \mathrm{~mol})$ of piperidine to a mixture of $0.4 \mathrm{~g}(0.0013 \mathrm{~mol})$ of furanone (1a) and $0.0026 \mathrm{~mol}$ of 4-cyanobenzaldehyde in $15 \mathrm{~mL}$ of methanol and stirring the mixture for $12 \mathrm{~h}$ at room temperature in a dark atmosphere. The mixture was then cooled and the precipitate was filtered off and washed with methanol on a filter and 2a1 was obtained as light-yellow crystals, which produced the desired compounds in good yield (up to 91\%). [33, 34]. All compounds obtained are soluble in THF and dichloromethane. The structures of these compounds were confirmed by HRMS and NMR (see the section 4 in supporting Information). 2a2-2a17 was prepared using the same procedure described for $2 \mathrm{a} 1$ above, and Compound 1a was used as the starting material. $2 \mathrm{~b} 1-2 \mathrm{~b} 3$ was prepared using the same procedure described for $2 \mathrm{a} 1$ above, and Compound $1 \mathrm{~b}$ was used as the starting material. 2a2-2a17 and 2b1$2 \mathrm{~b} 3$ were obtained as different color powder. The structural characterization was described in section 4 in supporting information. The selectivity of the aldol condensation was sufficiently high to generate Z-isomers that were confirmed by X-ray crystal analysis (CCDC deposit number: 2063013, Figure S1). [33]

\section{TDDFT (Time-Dependent Density Functional Theory) Calculations}

TDDFT calculations at the B3LYP/6-311++G(2d, p) level in DMSO were performed to predict the absorption and emission spectra for compounds 2a2, 2a6, 2a12 and $2 \mathbf{a 1 6}$ 
using Gaussian 09 package. Solvent effects were taken into account by employing the default IEFPCM model for DMSO. The differences between LUMO and HOMO molecular orbital energies at the ground state were used to calculate the maximum absorption wavelength. The energy differences between the optimized first excited state and the corresponding ground state were used to calculate the emission spectra by applying Franck-Condon principle. The calculated spectra were shown in Table S2.

\section{Results and discussion}

\subsection{Fluorescent properties of the novel rofecoxib analogues.}

The fluorescent properties of rofecoxib analogues (2a1-2a17, 2b1-2b3) were shown in Figure 2A-2B and Table S1. The emission wavelengths of rofecoxib analogues can be tuned by introducing various substituents leading to different lengths of the $\pi$ conjugation system. As shown in Figure 2B, it was observed that the more electrondonating substitutions on $\mathrm{C}-4$ of $\mathrm{Ar}^{3}$ ring showed more bathochromic emission in DMSO, indicating the formation of a D- $\pi$-A system. For example, compounds $\mathbf{2 a 6}$, 2a8, 2a12-2a17 and 2b1-2b3 with EDG (EDG-O for EDG on the oxygen atom and EDG-N for EDG on the nitrogen atom) at the ortho-position or para-position on the phenyl group $\left(\mathrm{Ar}^{3}\right)$ exhibited red shifts in their emission wavelengths, compared to the analogues with EWG (2a1-2a5). Also, the EDG-N substituted compounds 2a122a17 showed longer emission wavelengths, ranging from $649 \mathrm{~nm}$ to $680 \mathrm{~nm}$ as compared with EDG-O substituted compounds (2a6, 2a8 and 2b1-2b3). Among compounds $2 \mathbf{a 6}-\mathbf{2 a 8}, \mathbf{2 b 1 - 2 b 3}$, it was found that introduction of hydroxyl groups at the meta-position of the $\mathrm{Ar}^{3}$ ring led to blue-shifts of the emission wavelengths and 
decreases of their fluorescent intensities when compared with analogues whose hydroxyl groups are located at ortho or para-positions (Figure S2-S5), indicating that such a modification was not favorable for their spectral properties probably due to mainly the group's inductive effect on the meta-position of a benzene ring.
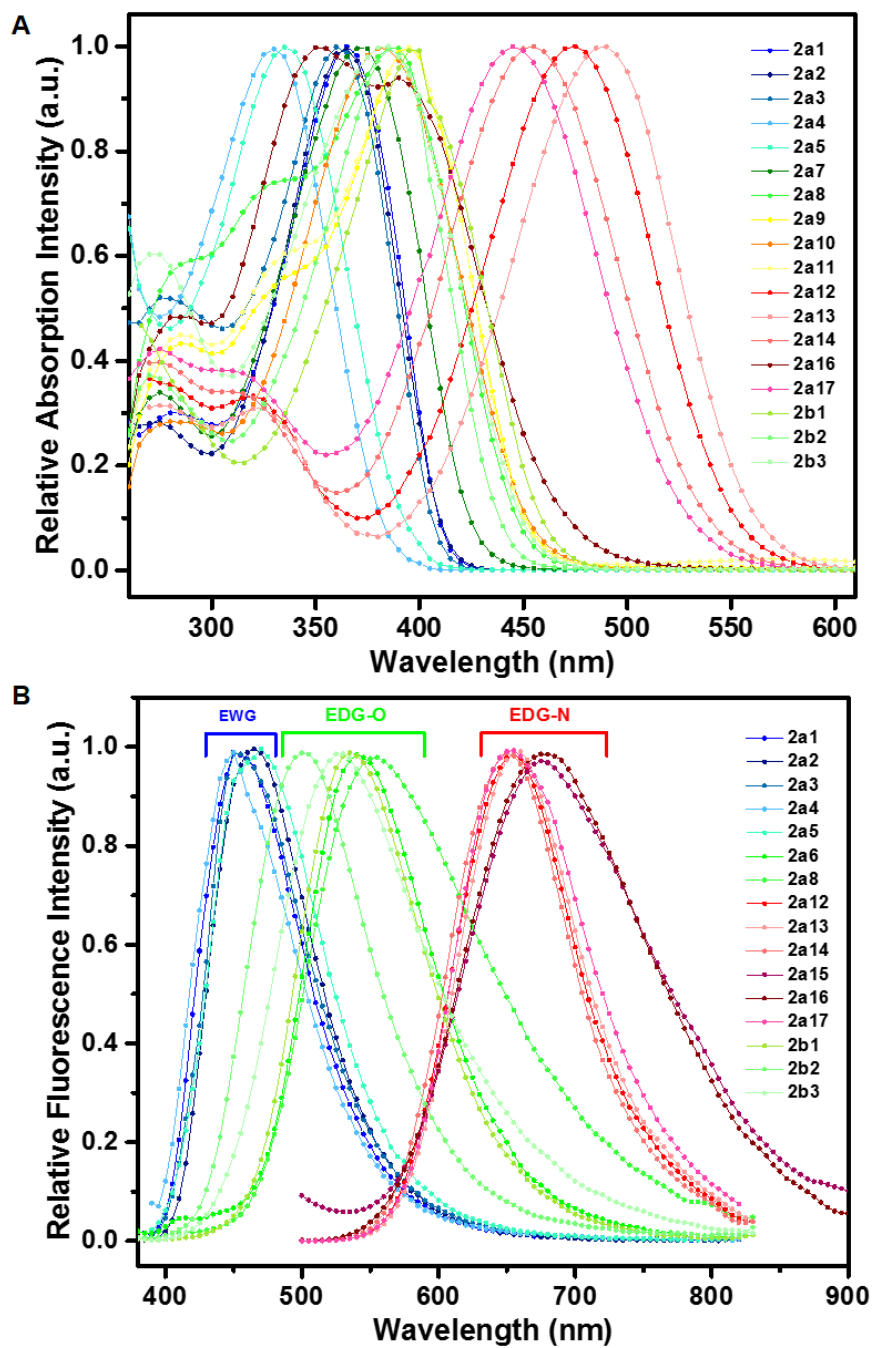

Figure 2. (A) The absorption spectra of compounds 2a1-2a5, 2a7-2a14, 2a16, 2a17, 2b1-2b3 in DMSO. (B) The emission spectra of compounds 2a1-2a6, 2a8, 2a12-2a16, 2b1-2b3. The compounds were divided into three groups termed EWG, EDG-O and EDG-N based on their maximum emission wavelength (Excited wavelength: $365 \mathrm{~nm}$, $100 \mu \mathrm{M})$. 


\subsection{Internal Charge Transfer (ICT) and Solvatochromic effect of $\mathbf{2 a 1 6}$}

Next, compound 2a16 was chosen to further investigate its spectral property in different solvents due to its near-red emission and excellent quantum yield. The UV-vis absorption spectra in different solvents such as toluene (PhMe), dichloromethane (DCM), tetrahydrofuran (THF), ethanol (EtOH), dimethyl sulfoxide (DMSO), were shown in Figure 3A, and the corresponding fluorescent data were summarized in Figure 3B. It was found that $\mathbf{2 a 1 6}$ showed two absorption bands between $340 \mathrm{~nm}$ and $390 \mathrm{~nm}$ in different solvents (Fig. 3A). The low energy absorption band originated from ICT (intramolecular charge transfer) transition, while the higher energy band ranging from 320-350 $\mathrm{nm}$ can be attributed to $\pi-\pi^{*}$ transitions of the benzene ring moiety. [35] Its maximum emission wavelength varied from $588 \mathrm{~nm}$ to $675 \mathrm{~nm}$ when the solvent changed from PhMe to $1.21 \%$ in DMSO. Meanwhile, the $\Phi_{\mathrm{f}}$ value of $\mathbf{2 a 1 6}$ was the highest at $28.5 \%$ in $\mathrm{PhMe}$ and much lower in other solvent such as DCM, EtOH and DMSO (Figure 3B). The emission intensity also decreased pronouncedly, which were mainly due to the non-radiative decay in polar solvents. [36] These results indicated that 2 a16 was strongly dependent on the solvent polarity. To have a better insight into the ICT process, quantum mechanical calculations were performed on compound $\mathbf{2 a 1 6}$ to study its ICT mechanism at the B3LYP/6-31++G(2d,p) level. The molecular orbitals of HOMO (highest occupied molecular orbital) and LUMO (lowest unoccupied molecular orbital) were depicted in Figure 3C and 3D. The ICT was evident that when excited the electrons will move from the electron-rich 2-morpholinophenyl group as the donor in HOMO to the methylsulfonylphenyl group as the receptor in LUMO. 

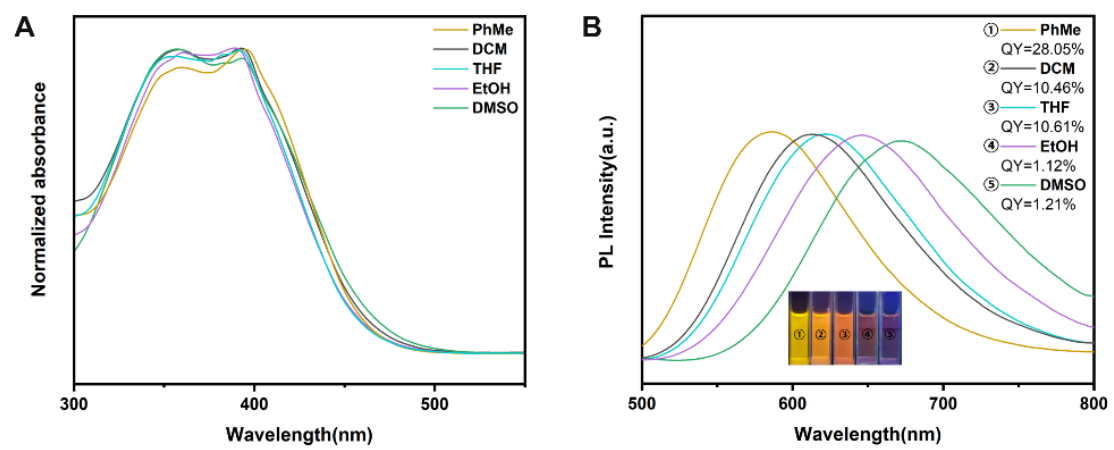

C

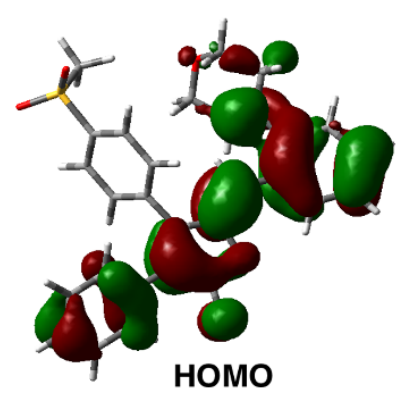

D

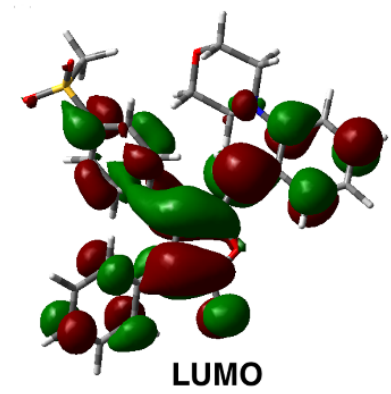

Figure 3. The UV-vis (A) and fluorescence spectra (B) of $2 \mathbf{a 1 6}$ in different solvents $(50.0 \mu \mathrm{M})$. The optimized structure of $2 \mathrm{a16}$ and its HOMO (C) and LUMO (D) at the B3LYP/6-31++G(2d,p) level.

\subsection{The summary of the structure and fluorescent properties relationship (SFR)}

Next, 10 analogues in Figure 4 were selected to illustrate the effect of substituent of $\mathrm{Ar}^{3}$ on fluorescent properties. It was found that the strong electron-donating substituents on the $\mathrm{C}-4$ position of $\mathrm{Ar}^{3}$ ring resulted in large red-shifts in the emission wavelengths for rofecoxib analogues because of the formation of a D- $\pi$-A system (Figure 4A). For example, as shown in Figure 4A and 4B, compared to $\mathbf{2 a 2}(\lambda \max =$ $482 \mathrm{~nm}$ ), it was found that the $\mathrm{N}$-substitutions on the $\mathrm{Ar}^{3}$ ring led to dramatic red-shifts in their emission wavelengths for compounds such as 2a13, 2a12, and 2a16, which have a maximum emission wavelength at $656 \mathrm{~nm}, 661 \mathrm{~nm}$, and $677 \mathrm{~nm}$, respectively (Figure 4B). In contrast, the emission maximum of $\mathbf{2 a 1}$ was blue-shifted to $453 \mathrm{~nm}$ by 
introduction of an electron-withdrawing methylsulfonyl group on the $\mathrm{Ar}^{3}$ ring (Figure 4B). Next, compounds 2a1-2a3, 2a6, 2a12 and 2a13 were used for analysis of the Hammett substituent constant for the para functional group on the $\mathrm{Ar}^{3}$ ring. Electronegativity and emission wavelength showed a positive correlation, as expected (Figure 4C). ${ }^{[37]}$ Furthermore, TDDFT (time-dependent density functional theory) calculations at the B3LYP/6-311++G(2d,p) level in DMSO were also performed to explain the absorption and emission spectra of compounds 2a2, 2a6, 2a12. The results confirmed that the energy gap between HOMO and LUMO of $\mathbf{2 a 2}$ was found out to be $3.29 \mathrm{eV}$. It was the largest of all three compounds, consistent with its shortest absorption wavelength. With the replacement with $\mathrm{OH}$ and $\mathrm{N}$-substituent group, the energy gaps of $2 \mathbf{a 6}$ and $2 \mathrm{a12}$ decreased to $3.10 \mathrm{eV}$ and $2.66 \mathrm{eV}$, respectively (Figure 4D), thus the increased absorption wavelengths. These results confirmed that there was a decrease in energy gas thus the bathochromic shift in the wavelength, which was in agreement with the spectral shift in the experimental absorption spectra. ${ }^{[38]}$ Taken together, these data proved the importance of the substituent on the $\mathrm{Ar}^{3}$ ring in tuning the spectral properties of the target compounds. Those SFR studies should provide a guideline for future design of rofecoxib-based fluorescent probes. 


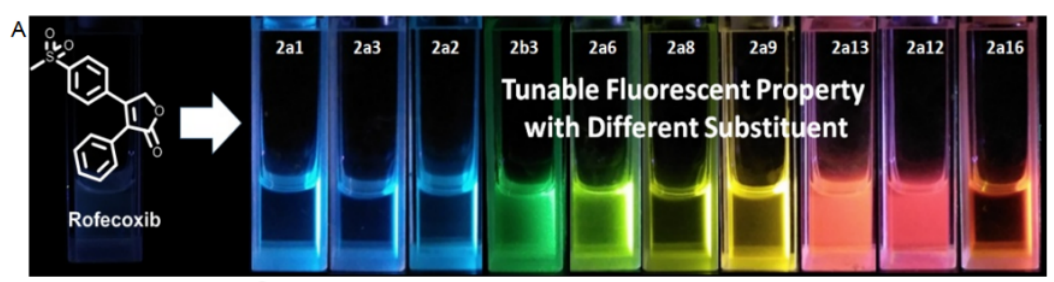

B

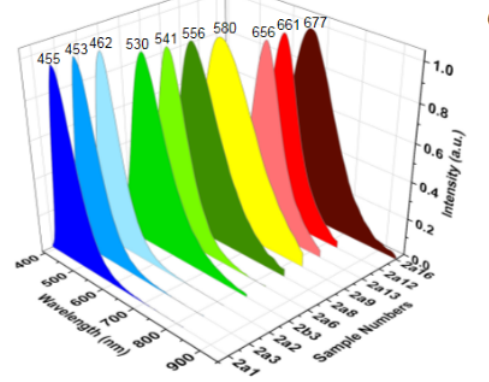

c

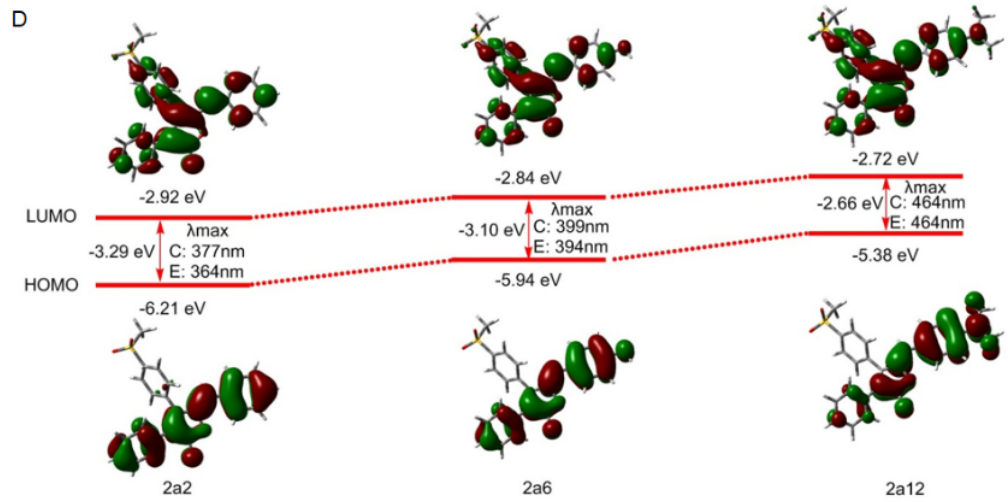

Figure 4. (A) structure-fluorescent properties relationship of rofecoxib analogues, the image was taken using a hand-held UV lamp (365 nm) as excitation. Stock solutions of them were made in DMSO $(100 \mu \mathrm{M})$. (B) Emission spectra of ten rofecoxib analogues are shown. (C) Correlation between emission wavelength and Hammett constant ( $\sigma \mathrm{p})$ of $\mathrm{R} 1$ substituents in rofecoxib analogues $\left(\mathrm{R}^{2}=0.84\right)$. (D) TDDFT calculations to explain absorption wavelength, and comparison between Calculation value (Cal) and Experimental (Exp) value of maximum absorption wavelength.

\subsection{AIE properties of $\mathbf{2 a 1 6}$}

Conventional fluorophores featured with $\pi$-planar structures usually suffer from serious self-quenching in the aggregated state, poor photostability, and small Stokes' 
shift. In contrast to conventional aggregation-caused quenching (ACQ) fluorophores, AIEgens are featured with high emission efficiency in the aggregated state, which could be useful in various sensing applications with advantages of high signal-to-noise ratio, strong photostability, and large Stokes's shift. The AIE behavior of compound 2a16 was investigated next due to the existence of half tetraphenylethene moeity in its structure which could endow the compound with AIE activity. It was shown that $\mathbf{2 a 1 6}$ had weak fluorescence in DMSO (Figure 2 and 5A). When the water fraction was increased to $60-80 \%$, the fluorescence intensity began to increase and reached a maximum value at $80 \%$ water fraction (Figure $5 \mathrm{~A}$ and $5 \mathrm{~B}$ ). Furthermore, the fluorescence quantum efficiency $\left(\Phi_{\mathrm{f}}\right)$ of $\mathbf{2 a 1 6}$ in pure DMSO solution was merely $0.4 \%$, which was enhanced to $31 \%$ at $60 \%$ water fraction and $94 \%$ in the solid powder (Table S1 and Figure 5B), 235-fold increase. Such experimental data demonstrated that 2 a16 could aggregate in the non-solvent and showed a significant AIE effect. Such optical properties made $2 \mathbf{a 1 6}$ an ideal candidate for the development of anti-photobleaching particles for various imaging applications. ${ }^{[39-42]}$
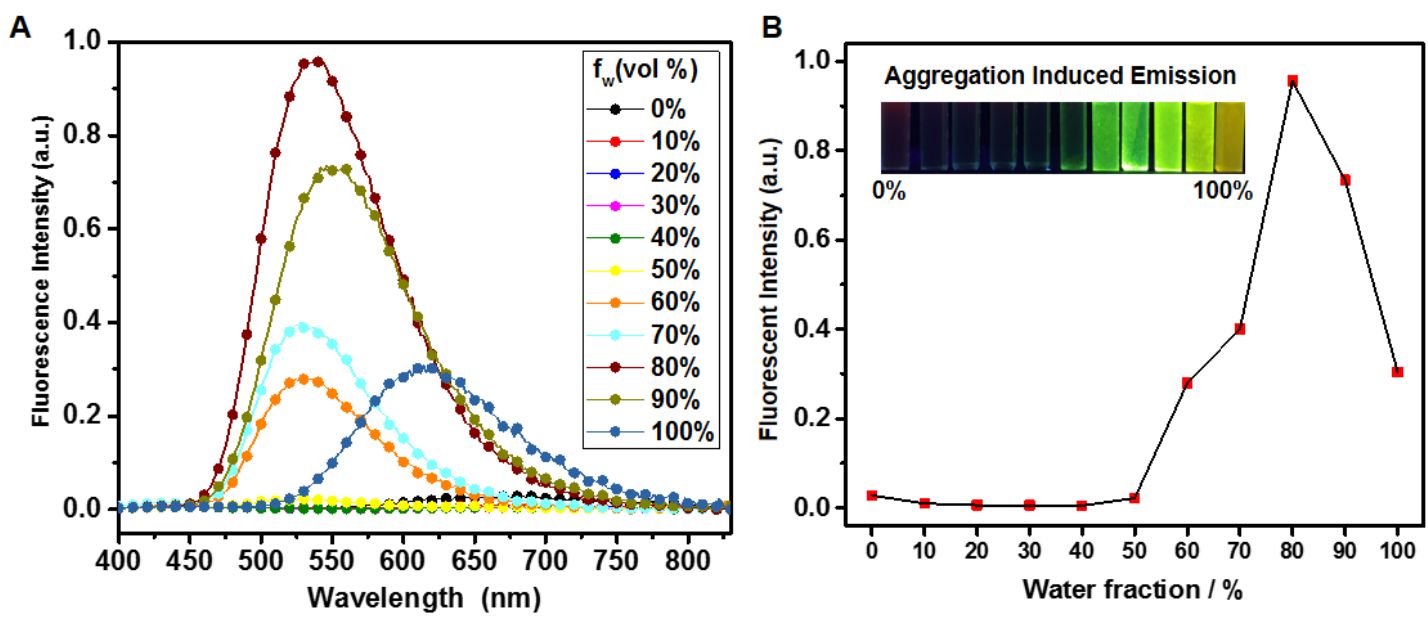
Figure 5. (A) Fluorescence spectra in $\mathrm{DMSO} / \mathrm{H}_{2} \mathrm{O}$ with different water fractions. (B)

Plot of the maximum peak fluorescence intensities of $2 \mathbf{a 1 6}$ in $\mathrm{DMSO} / \mathrm{H}_{2} \mathrm{O}$ with different water fractions. Inset: fluorescence images (Excited wavelength: 365 nm, 100 $\mu \mathrm{M})$

\subsection{Mechanochromic properties of $\mathbf{2 a 1 6}$}

A

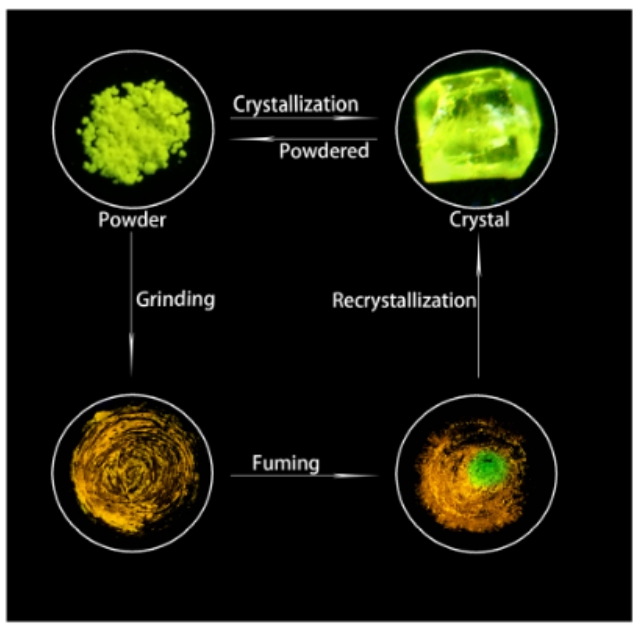

C

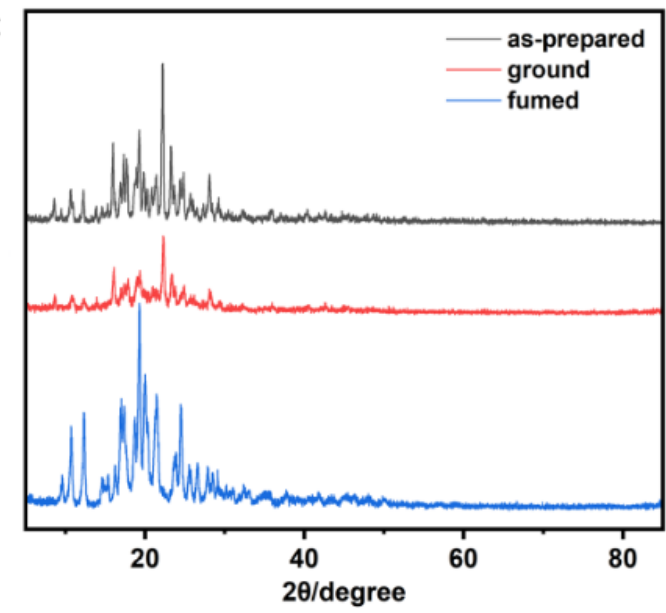

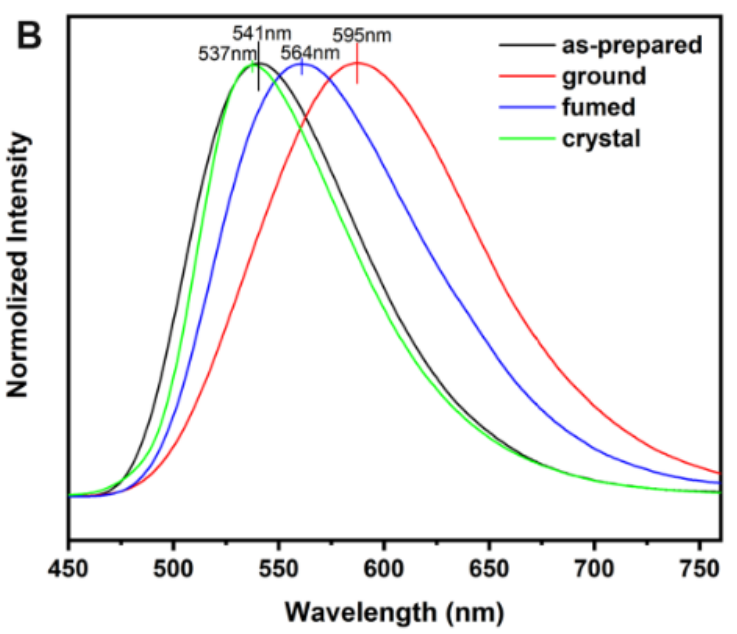

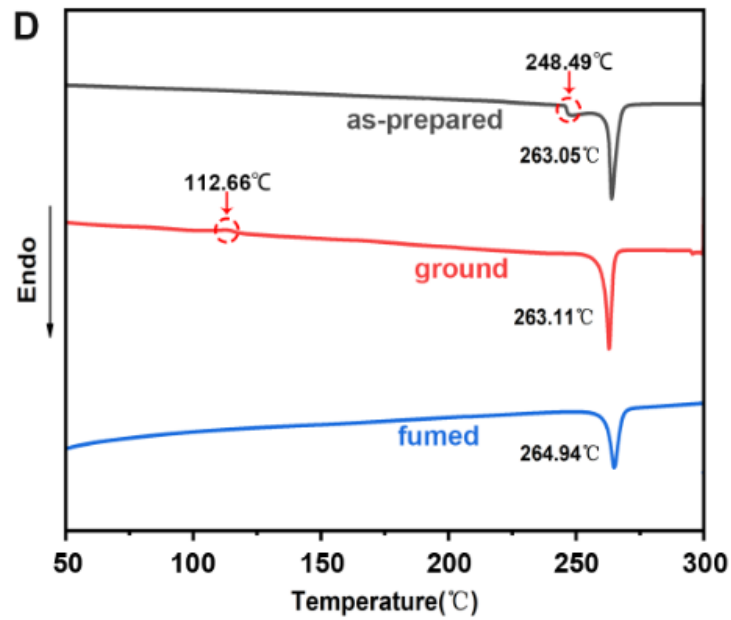

Figure 6. (A) Optical images taken using a hand-held UV lamp (365 nm) as excitation.

(B) Fluorescent spectra of the as-prepared (pristine) green powder, crystal, ground orange powder and fumed powder (Excited wavelength: $365 \mathrm{~nm}$ ). (C) PXRD curves of 
the pristine green powder, ground orange powder and fumed green powder. (D) DSC curves of the pristine powder, orange powder and fumed powder.

Mechanochromism is the phenomenon of change in color due to mechanical grinding or pressing of a solid sample, and the subsequent reversion to its original color through treatment such as heating or recrystallization. Photoluminescent compounds that possess mechanochromic properties in their solid-state emissions can provide unique recording or sensing materials that involve luminescence detection. Following the AIE investigation of $\mathbf{2 a 1 6}$, its mechanochromic property was further investigated in this section. As shown in Figure 6A, 2 a16 exhibited two-color changing mechanochromic luminescence $(\mathrm{MCL})$ behavior in response to the external stimuli. The pristine powder of 2 a16 emitted green luminescence under a $365 \mathrm{~nm}$ UV lamp and the maximum emission wavelength $(\lambda \max )$ was $541 \mathrm{~nm}$ (Figure 6A and 6B). After the pristine powder was ground using a pestle on a mortar at room temperature, the ground powder exhibited orange emission $(\lambda \max =595 \mathrm{~nm})$. The orange state can revert back to the green with emission $(\lambda \max =564 \mathrm{~nm})$ upon fuming by acetone vapor (Figure 6A and 6B). To investigate the mechanism of mechanochromic properties, powder X-ray diffraction (PXRD) and differential scanning calorimetry (DSC) measurements were carried out. As shown in Figure 6C, the pristine powder of 2 a16 showed intense and sharp peaks, suggesting the crystalline state. In contrast, the PXRD curve obtained by grinding did not exhibit obvious peak, indicating that the pristine powder had changed from the crystalline state to an amorphous one after mechanical stress. ${ }^{[43-46]}$ Upon fuming the ground powder in acetone, some new peaks appeared. The partly different 
PXRD curves of fumed powder compared to those of the pristine powder demonstrated the change from one crystalline phase to another, which was also consistent with their different emission spectral between pristine powder and fumed powder (Figure 6B). Moreover, the DSC curves revealed that the melting point of $2 \mathbf{a 1 6}$ was about $263^{\circ} \mathrm{C}$ (Figure 6D). The pristine sample showed an endothermic transition peak at $248^{\circ} \mathrm{C}$, corresponding to phase transition as opposed to cold crystallization with a melting point of $263^{\circ} \mathrm{C} .{ }^{[42]} \mathrm{By}$ contrast, DSC curve of the ground sample showed a new exothermic peak around $116^{\circ} \mathrm{C}$ next to the melt peak at $264^{\circ} \mathrm{C}$, which was absent in the pristine sample and the fumed sample. ${ }^{[44]}$ This broad exothermic peak could be ascribed to cold crystallization transition, which revealed that the amorphous phase of the ground sample was a metastable state.

\subsection{The mechanistic study on $M C L$}

Based on the results above, it was hypothesized that the two-color mechanochromic luminescence of $\mathbf{2 a 1 6}$ was ascribed to the force-induced transformation of the molecular packing. Therefore, it was of great significance to study the mechanism on MCL of $2 \mathbf{a 1 6}$ by analyzing the single crystal of the compound. To our delight, a single crystal of 2a16 was obtained and analyzed by X-ray diffraction (Figure 7A). The crystal data and the collection of parameters were summarized in the Supporting Information (see crystal data of 2a16). Compound 2 a16 packed in the monoclinic crystal system, from which two representative tetramers were extracted to illustrate its intermolecular packing. As shown in Figure 7A, the phenyl group (Ring $A=A r^{1}$ ) and 4(methylsulfonyl)phenyl group (Ring $\mathrm{B}=\mathrm{Ar}^{2}$ ) adopted a twisted conformation to 
connect to the lactone ring, with their dihedral angles as $33.91^{\circ}$ and $57.26^{\circ}$, respectively. The other phenyl group (Ring $\mathrm{C}=\mathrm{Ar}^{3}$ ) adopted a less twisted conformation to connect to the double bond with the dihedral angle as $19.58^{\circ}$. The dihedral angles among the phenyl rings were calculated to be $59.46^{\circ}, 53.21^{\circ}$, and $57.86^{\circ}$ (Figure 7A). It is interesting to note that two $\mathrm{C}-\mathrm{H} \cdots \mathrm{O}$ interactions existed between the oxygen atom of the morpholine ring and the $\mathrm{C}-\mathrm{H}$ of methylsulfonyl group and also another $\mathrm{C}-\mathrm{H}$ of the morpholine ring (2.472 and $2.552 \AA$, respectively) (Figure 7B). In addition, ene $\mathrm{C}-\mathrm{H} \cdots \pi$ interaction was formed between the $\mathrm{C}-\mathrm{H}$ of the phenyl ring and the double bond adjacent to the lactone ring (2.872 $\AA$ ). Two more $\mathrm{C}-\mathrm{H} \cdots \mathrm{O}$ interactions existed between the oxygen atom of the ketone group and two $\mathrm{C}-\mathrm{H}$ of the neighboring phenyl groups (2.622 and $2.537 \AA$, respectively) (Figure S6). Such interactions between adjacent molecules greatly restricted their rotations and efficiently suppressed their nonradiative relaxation therefore enabled the molecules to emit intense emission in crystals. It is worth mentioning that the cooperation of those multiple interactions made it possible for the molecules to arrange in a slipped parallel manner within the crystals along the a, b, and c directions with intermolecular distances of 22.337, 6.324, and 18.417 $\AA$, respectively (Figure 7C). Such tight packing greatly favored the AIE effect and mechanochromic luminescence of compound $2 \mathbf{a 1 6}$ as observed experimentally (Figure 7C). 

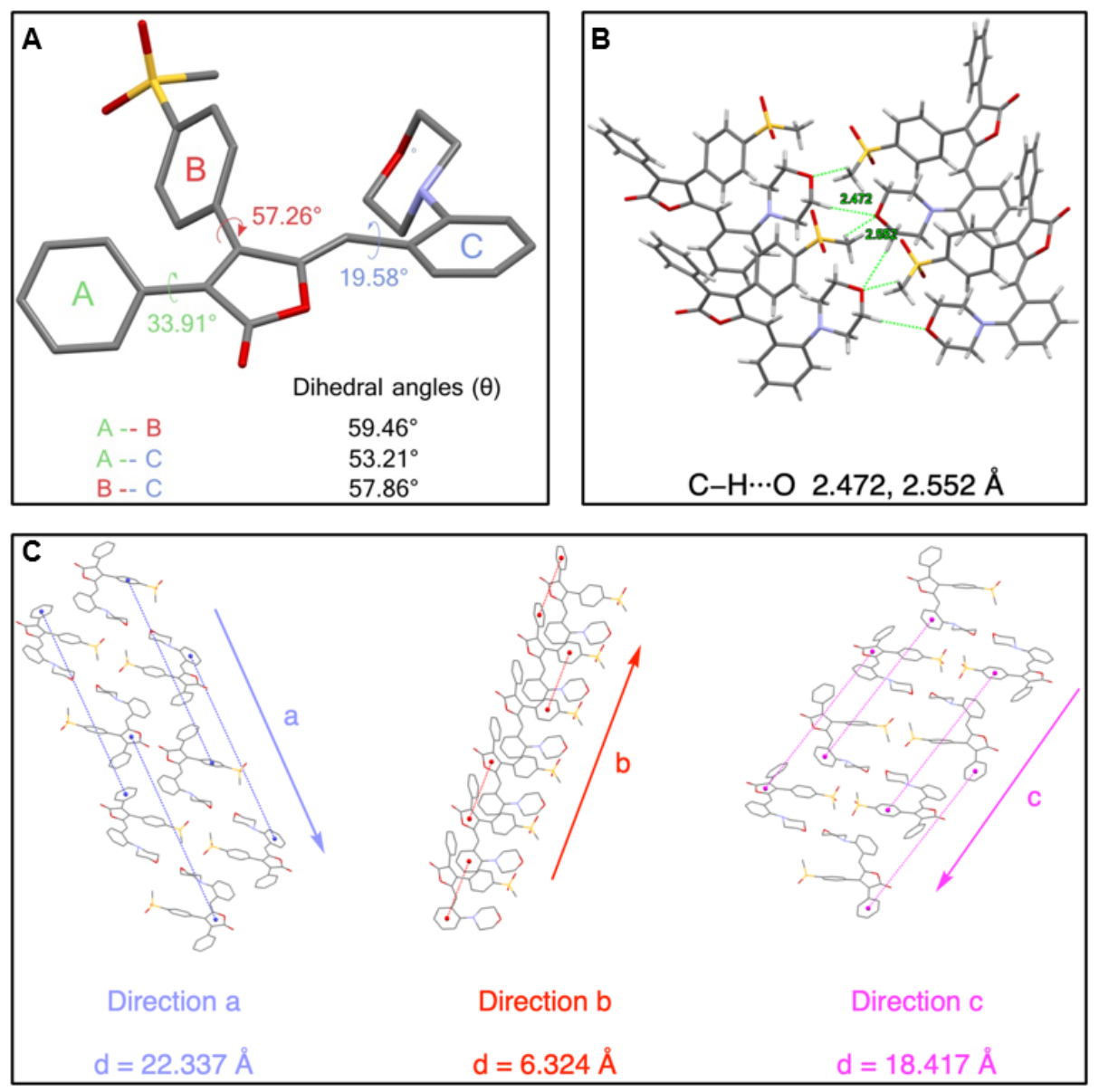

Figure 7. (A) Single crystal structure of 2a16. (B) Two dimers extracted from the single-crystal structure of $\mathbf{2 a 1 6}$ with bond lengths. (C) Molecular stacking structures along the a, b, and c directions in $\mathbf{2 a 1 6}$ crystals.

\subsection{2 a16 interactions with COX-2 enzyme}

From the results above, some of the designed rofecoxib analogues have been demonstrated to show the AIE characteristic with red emission, mechanochromism and large Stokes shift. Among them, compounds 2a12-2a17 appeared to be the most promising due to their high quantum yields in powder and longer emission (Table S1). They were first selected for COX-2 molecular docking to study their binding modes with COX-2 enzyme. ${ }^{[47,48]}$ The binding modes of compounds 2a12-2a17 largely 
resembled that of rofecoxib, as expected. It was shown in Figure 8A by using compound $2 \mathbf{2 1 6}$ as an example. It was observed that the methyl sulfone moiety of both rofecoxib (blue) and 2 a16 (pink) was in one part of the active site formed by residues Arg513, His90, Gln192, and Phe518. The phenyl ring attached to the lactone ring in rofecoxib fit well in another part of the active site formed by Trp387, Tyr385, and Ser530. However, the same phenyl ring in $\mathbf{2 a 1 6}$ was flipped to fit in the sub-pocket formed by residues Glu524, Arg120, and Leu531. It so happened that it could make room for the larger moiety of phenyl morpholine introduced on the lactone ring. $\mathbf{2 a 1 6}$ could fit into the active site and more interactions with the surrounding residues were introduced with the additional fragments. These docking results provided a theoretical explanation at the molecular level for the following imaging studies at the cellular level. Before cell imaging experiments, the potential cytotoxicity of rofecoxib analogues was evaluated by using MTT assay and low cytotoxicity of the analogues was observed (Figure S7 and S8). Subsequently, the confocal fluorescence imaging experiments were performed in live HeLa cells. Three rofecoxib analogues successfully penetrated the cell membranes of HeLa cells. As demonstrated in Figure S9, all of them were cell permeable and were suitable for live cell imaging. In particular, compound $2 \mathbf{2} 16$ exhibited a bright fluorescent image in live cells.

\subsection{Fluorescence imaging of COX-2 overexpressed cells}

To assess the COX-2 binding ability of $\mathbf{2 a 1 6}$ in COX-2 overexpressed cells, fluorescence images were taken after it was incubated with different cells. According to the western blotting assay, it was found that the expression of COX-2 was 
significantly high in HeLa cells but not in RAW264.7 cells. (Figure 8B). As expected, after incubating with 2a16, bright red fluorescence was observed in HeLa cells, which was not observed in RAW264.7 cells (Figure 8C). It indicated that 2 a16 was able to distinguish cancer cells from normal cells by labeling overexpressed COX-2 in cancer cells.

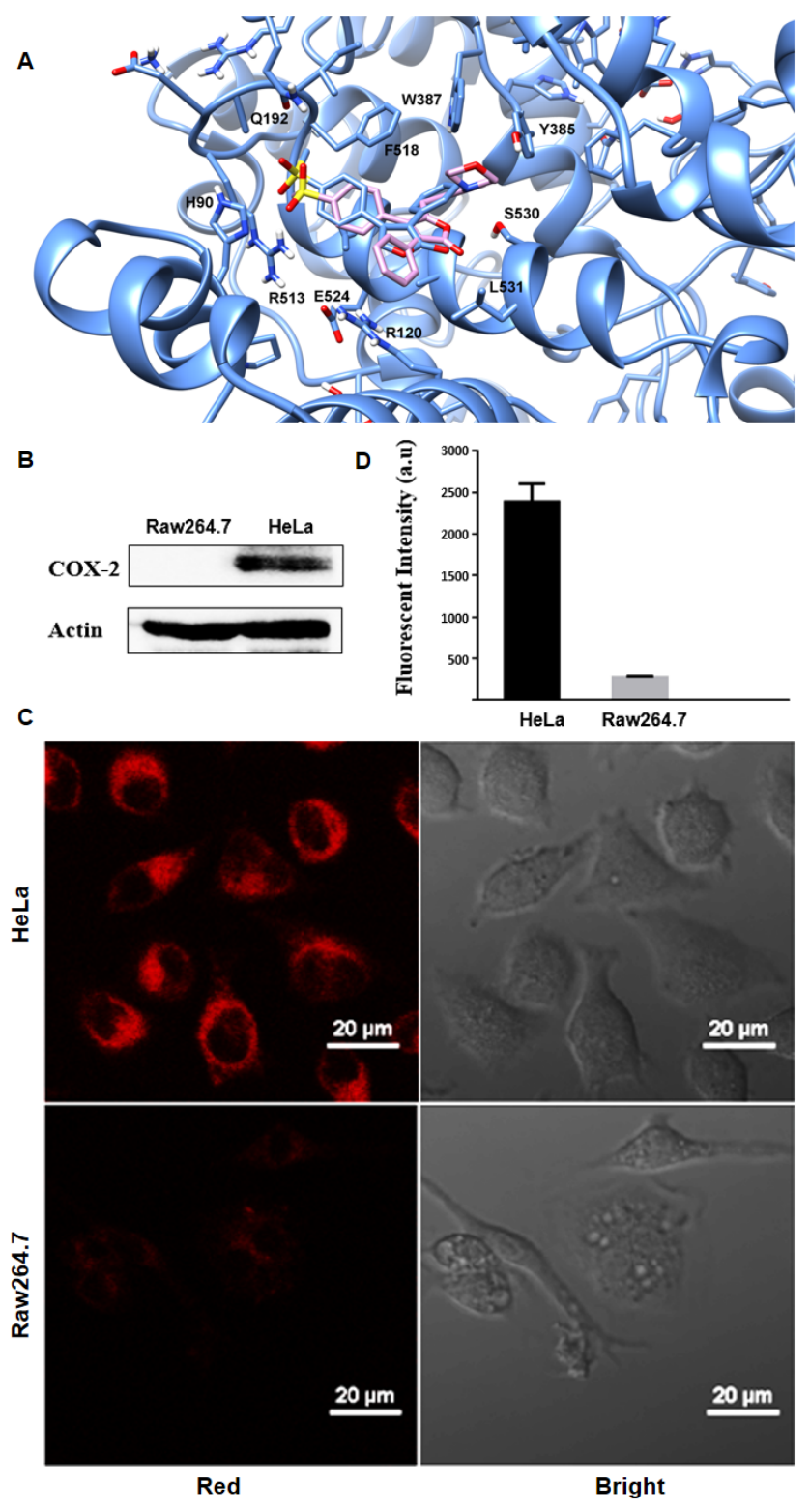

Figure 8. (A) COX-2 docking study of rofecoxib analogues. The binding modes of rofecoxib (blue) and compound 2a16 (pink) at the active site of COX-2. The 
experimental crystallographic structure of COX-2 complex was retrieved from the Protein Data Bank (PDB: 3ln1) (B) Western blot showing COX-2 expression level in RAW264.7 and HeLa cells. (C) Fluorescence images of RAW264.7 and HeLa cell lines with confocal microscopy. All cells were treated with 2 a16 $(2 \mu \mathrm{M})$ for $2 \mathrm{~h}$. (Magnification: $\times 40 ; \lambda$ ex: $405 \mathrm{~nm}$; Red, $\lambda$ em: 570-1000 nm). Images are representative of triplicate experiments ( $n=3$, see Figure S10). (D) Quantitative image analysis of the average total fluorescence intensity of HeLa cell and RAW264.7 cell.

\subsection{Fluorescence imaging of cancer tissues}

The results at the cellular level are not necessarily reproducible in tissue samples because of the variability and complexity of clinical samples. Therefore, to assess the potential of $2 \mathbf{a 1 6}$ in clinical applications as an imaging agent, esophageal cancer tissues were used to study whether $\mathbf{2 a 1 6}$ can distinguish the cancer tissue from the adjacent normal tissues because it was reported that there are an estimated 572,034 new esophageal cancer cases and 508, 585 deaths every year worldwide. Consistent with the previous research, it was found that both the mRNA and the COX-2 protein were upregulated in human esophageal squamous cell carcinoma (ESCC) (Figure 9A and 9B). ${ }^{[49-51]}$, As shown in Figure 9C, after incubated with 2a16, bright red fluorescence was observed in ESCC tissues, but weak fluorescence in adjacent tissues. These results are consistent with the COX-2 expression level in the cancer tissues. These results strongly suggested that $\mathbf{2 a 1 6}$ had the potential to be developed as new COX-2 probes for detecting cancer tissues in clinical applications. 


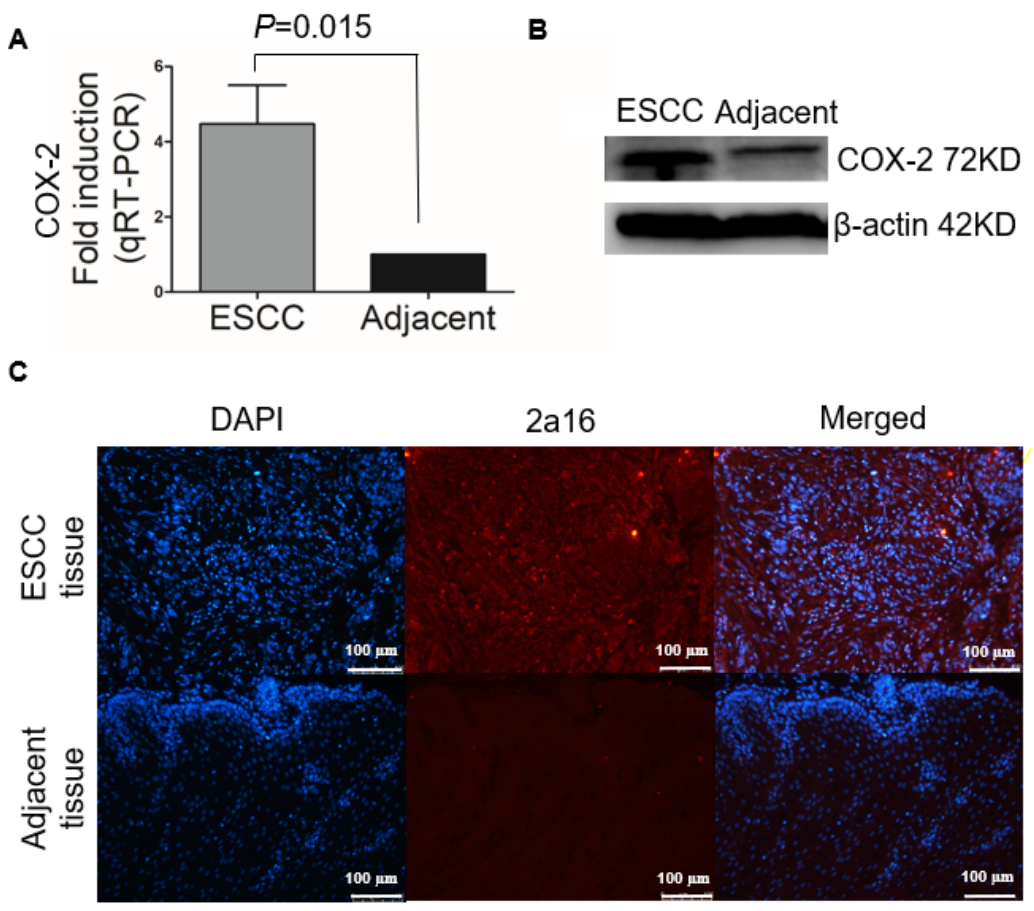

Figure 9. (A) qRT-PCR for mRNA analysis of COX-2 in human ESCC and adjacent tissue. (B) Western blot for protein analysis of COX-2 in human ESCC and adjacent tissue. (C) Fluorescence images of paraffin embedded section of human ESCC and adjacent tissue with inverted fluorescence microscope. All sections were treated with $2 \mathrm{a} 16(10 \mu \mathrm{M})$ for $0.5 \mathrm{~h}$.

\section{Conclusions}

In summary, we developed new COX-2 fluorescent probes using rofecoxib as the template. Some of the rofecoxib analogues designed are empowered with fluorescent characteristics, such as red emission, AIE property and mechanochromic behavior. Of them, 2 a16 was the most promising and it was shown to bind to the COX-2 enzyme over-expressed in HeLa cancer cells, resulting in bright red fluorescence imaging, but it will not light up the normal cells with minimal COX-2 expression. More importantly, in clinical esophageal cancer tissue samples, 2a16 was able to distinguish the cancer 
tissues from adjacent normal tissues by lighting up the COX-2 enzyme specifically. It paved the way for $\mathbf{2 a 1 6}$ to be developed as a cancer imaging agent, and even a cancer diagnostic agent.

\section{Conflicts of interest}

There are no conflicts to declare.

\section{CRediT authorship contribution statement}

Lijun Xie: Conceptualization, Investigation, Writing original draft. Renfu Li: Methodology, Investigation. Biyun Zheng: Investigation. Zuoxu Xie: Formal analysis. Xuefen Fang: Resources. Yanqi Wan: Crystal study of 2a16. Greg Cuny: Writing review. Zhenli Li: Computational study. Bin Lin: Computational study \& polishing the manuscript. Xueyuan Chen: Supervision. Ming Hu: Writing review \& editing, Supervision, Funding acquisition.

\section{Acknowledgments}

This work was supported by The National Institutes of Health GM070737 and Texas CPRIT RP180863 to MH, the Strategic Priority Research Program of the CAS (XDB20000000), the NSFC (Nos. 51872048, 21975257, 21771185), the NSF of Fujian Province (No. 2019I0029) to XC, NSFC (No. 21406143) to BL. LX spent time in MH's lab initiating this research, which was then completed when LX returned to his own lab in collaboration with $\mathrm{XC}$. BL also would like to acknowledge the faculty startup funding from Shenyang Pharmaceutical University.

\section{Abbreviations}


COX-2, cyclooxygenase-2; EWG, electron withdrawing group; EDG, electron donating group; AIE, aggregation induced emission; SFR, structure fluorescent properties relationship; TLC, thin layer chromatography; DMF, N, NDimethylformamide; THF, tetrahydrofuran; DMSO, dimethyl sulfoxide; DCM, dichloromethane; EtOH, ethyl Alcohol (Ethanol); D- $\pi$-A system, donor- $\pi$-acceptor; TDDFT, time-dependent density functional theory; NIR, near infrared; QY, quantum yields. PXRD, powder x-ray diffraction; DSC, differential scanning calorimetry; SXRD, single crystal x-ray diffraction; MCL, mechanochromic luminescence. ICT, internal charge transfer.

\section{AUTHOR INFORMATION}

Corresponding Authors

*E-mail: mhu4@uh.edu or xchen@fjirsm.ac.cn or randybinlin@gmail.com

\section{Author Contributions}

\#L.X., R.L. and B.Z. contributed equally. 


\section{References}

[1] Siegel, R. L.; Miller, K. D.; Jemal, A., Cancer Statistics, 2017. CA Cancer J. Clin., 2017, 67, 7-30.

[2] DeSantis, C. E.; Siegel, R. L.; Sauer, A. G.; Miller, K. D.; Fedewa, S. A.; Alcaraz, K. I.; Jemal, A., Cancer statistics for African Americans, 2016: Progress and opportunities in reducing racial disparities. CA Cancer J. Clin., 2016, 66, 290-308.

[3] Subbaramaiah, K.; Dannenberg, A. J., Cyclooxygenase 2: a molecular target for cancer prevention and treatment. Trends Pharmacol. Sci., 2003, 24, 96-102.

[4] Nath, S.; Roy, L. D.; Grover, P.; Rao, S.; Mukherjee, P., Mucin 1 Regulates Cox-2 Gene in Pancreatic Cancer. Pancreas., 2015, 44, 909-917.

[5] Wang, D.; Dubois, R. N., The role of COX-2 in intestinal inflammation and colorectal cancer. Oncogene., 2010, 29, 781-788.

[6] Echizen, K.; Hirose, O.; Maeda, Y.; Oshima, M., Inflammation in gastric cancer: Interplay of the COX-2/prostaglandin E2 and Toll-like receptor/MyD88 pathways. Cancer Sci., 2016, 107, 391-397.

[7] Uddin, M. J.; Crews, B. C.; Blobaum, A. L.; Kingsley, P. J.; Gorden, D. L.; McIntyre, J. O.; Matrisian, L. M.; Subbaramaiah, K.; Dannenberg, A. J.; Piston, D. W.; Marnett, L. J., Selective visualization of cyclooxygenase-2 in inflammation and cancer by targeted fluorescent imaging agents. Cancer Res., 2010, 70, 3618-3627. 
[8] Uddin, M. J.; Crews, B. C.; Huda, I.; Ghebreselasie, K.; Daniel, C. K.; Marnett, L. J., Trifluoromethyl fluorocoxib a detects cyclooxygenase-2 expression in inflammatory tissues and human tumor xenografts. ACS Med. Chem. Lett., 2014, 5, 446-450.

[9] Zhang, H.; Fan, J.; Wang, J.; Zhang, S.; Dou, B.; Peng, X., An off-on COX-2specific fluorescent probe: targeting the Golgi apparatus of cancer cells. J. Am. Chem. Soc., 2013, 135, 11663-11669.

[10] Zhang, H.; Fan, J.; Wang, J.; Dou, B.; Zhou, F.; Cao, J.; Qu, J.; Cao, Z.; Zhao, W.; Peng, X., Fluorescence discrimination of cancer from inflammation by molecular response to COX-2 enzymes. J. Am. Chem. Soc., 2013, 135, 17469-17475.

[11] Bhardwaj, A.; Kaur, J.; Wuest, F.; Knaus, E. E., Fluorophore-labeled cyclooxygenase- 2 inhibitors for the imaging of cyclooxygenase- 2 overexpression in cancer: synthesis and biological studies. ChemMedChem., 2014, 9, 109-116.

[12] Schuller, H. M.; Kabalka, G.; Smith, G.; Mereddy, A.; Akula, M.; Cekanova, M., Detection of overexpressed COX-2 in precancerous lesions of hamster pancreas and lungs by molecular imaging: implications for early diagnosis and prevention. ChemMedChem., 2006, 1, 603-610.

[13] Kim, H. S.; Park, T.; Ren, W. X.; Lim, J. Y.; Won, M.; Heo, J. S.; Lee, S. G.; Kim, J. S., COX-2 targeting indomethacin conjugated fluorescent probe. Dyes. Pigment., 2018, 150, 261-266. 
[14] Uddin, M. J.; Crews, B. C.; Ghebreselasie, K.; Marnett, L. J., Design, synthesis, and structure-activity relationship studies of fluorescent inhibitors of cycloxygenase-2 as targeted optical imaging agents. Bioconjug Chem., 2013, 24, 712-723.

[15] Gurram, B.; Zhang, S.; Li, M.; Li, H.; Xie, Y.; Cui, H.; Du, J.; Fan, J.; Wang, J.; Peng, X., Celecoxib Conjugated Fluorescent Probe for Identification and Discrimination of Cyclooxygenase-2 Enzyme in Cancer Cells. Anal Chem., 2018, 90, $5187-5193$

[16] Liu, C.; Yang, C.; Lu, L.; Wang, W.; Tan, W.; Leung, C. H.; Ma, D. L., Luminescent iridium(iii) complexes as COX-2-specific imaging agents in cancer cells. Chem. Commun., 2017, 53, 2822-2825.

[17] Zhang, Q.; Han, Z.; Tao, J.; Zhang, W.; Li, P.; Tang, L.; Gu, Y., A novel nearinfrared fluorescent probe for monitoring cyclooxygenase- 2 in inflammation and tumor. J. Biophotonics., 2018, 11, e201700339.

[18] Bhardwaj, A.; Kaur, J.; Sharma, S. K.; Huang, Z.; Wuest, F.; Knaus, E. E., Hybrid fluorescent conjugates of COX-2 inhibitors: search for a COX-2 isozyme imaging cancer biomarker. Bioorg. Med. Chem. Lett., 2013, 23, 163-168.

[19] Zhang, H.; Fan, J.; Wang, K.; Li, J.; Wang, C.; Nie, Y.; Jiang, T.; Mu, H.; Peng, X.; Jiang, K., Highly sensitive naphthalene-based two-photon fluorescent probe for in situ real-time bioimaging of ultratrace cyclooxygenase-2 in living biosystems. Anal Chem., 2014. 86, 9131-9138. 
[20] Lee, J. Y., Kim, H. S., Jangili, P., Kang, H. G., Sharma, A., Kim, J. S., Fluorescent Probe for Monitoring Hydrogen Peroxide in COX-2- Positive Cancer Cells. ACS Appl. Bio Mater. 2020, doi.org/10.1021/acsabm.0c01135.

[21] Wang, X. L., Wang, L. Y., Xie, L. J., Xie, Z. X., Li, L., Bui, D., Yin, T. J., Gao, S., Hu, M. Design and Synthesis of a Novel NIR Celecoxib-Based Fluorescent Probe for Cyclooxygenase-2 Targeted Bioimaging in Tumor Cells. Molecules., 2020, 25, 4037-4049.

[22] Chen, Y., Design and construction of COX-2 specific fluorescent probes. Mol. Cell. Probes., 2019, 48, 101472.

[23] Cao, X. Z.; Gao, T., Dong, J.; Jiang, X. C.; Zou, H.; Liu, T. T.; Yu, K. Q.; Zeng, W. B., An AIE-based self-assembled fluorescent probe for COX-2 imaging. New J. Chem., 2019, 43, 7874-7880.

[24] Xiong, J.; Wang, K.; Yao, Z.; Zou, B.; Xu, J.; Bu, X.-H., Multi-Stimuli-Responsive Fluorescence Switching from a Pyridine-Functionalized Tetraphenylethene AIEgen. ACS Appl. Mater. Inter., 2018, 10, 5819-5827.

[25] La, D. D.; Bhosale, S. V.; Jones, L. A.; Bhosale, S. V., Tetraphenylethylene-Based AIE-Active Probes for Sensing Applications. ACS Appl. Mater. Inter., 2018, 10, $12189-12216$.

[26] Thooft, A. M.; Cassaidy, K.; VanVeller, B., A Small Push-Pull Fluorophore for Turn-on Fluorescence. J. Org. Chem., 2017, 82, 8842-8847. 
[27] Dar, A. H.; Gowri, V.; Gopal, A.; Muthukrishnan, A.; Bajaj, A.; Sartaliya, S.;

Selim, A.; Ali, M. E.; Jayamurugan, G., Designing of Push-Pull Chromophores with Tunable Electronic and Luminescent Properties Using Urea as the Electron Donor. $J$. Org. Chem., 2019, 84, 8941-8947.

[28] Ahn, M.; Kim, M.-J.; Wee, K.-R., Electron Push-Pull Effects in 3,9-Bis(p-(R)diphenylamino)perylene and Constraint on Emission Color Tuning. J. Org. Chem., 2019, 84, 12050-12057.

[29] Yamaguchi, Y.; Matsubara, Y.; Ochi, T.; Wakamiya, T.; Yoshida, Z.-i., How the $\pi$ Conjugation Length Affects the Fluorescence Emission Efficiency. J. Am. Chem. Soc., 2008, 130, 13867-13869.

[30] Xu, S.; Evans, R. E.; Liu, T.; Zhang, G.; Demas, J. N.; Trindle, C. O.; Fraser, C. L., Aromatic Difluoroboron $\beta$-Diketonate Complexes: Effects of $\pi$-Conjugation and Media on Optical Properties. Inorg Chem., 2013, 52, 3597-3610.

[31] Vendrell, M.; Zhai, D.; Er, J. C.; Chang, Y. T., Combinatorial strategies in fluorescent probe development. Chem. Rev., 2012, 112, 4391-420.

[32] Kobayashi, H.; Ogawa, M.; Alford, R.; Choyke, P. L.; Urano, Y., New strategies for fluorescent probe design in medical diagnostic imaging. Chem. Rev., 2010, 110, 2620-2640. 
[33] Liu, G. Z.; Xu, H. W.; Wang, P.; Lin, Z. T.; Duan, Y. C.; Zheng, J. X.; Liu, H. M., Stereoselective synthesis and anti-proliferative effects on prostate cancer evaluation of 5-substituted-3,4-diphenylfuran-2-ones. Eur. J. Med. Chem., 2013, 65, 323-336.

[34] Lei, M.; Gan, X.; Zhao, K.; Chen, A.; Hu, L., Synthesis of 3,4-disubstituted-furan2(5H)-one derivatives by Suzuki-Miyaura reaction. Tetrahedron., 2015, 71, 33253332.

[35] Zhu, Y. Z.; Du, W.; Zhang, M. Z.; Xu, Y.; Song, L. L.; Zhang, Q.; Tian, X. H.; Zhou, H. P.; Wu, J. Y.; Tian, Y. P., A series of water-soluble A- $\pi-\mathrm{A}^{\prime}$ typological indolium derivatives with two-photon properties for rapidly detecting $\mathrm{HSO}_{3}{ }^{-} / \mathrm{SO}_{3}{ }^{2-}$ in living cells. J. Mater. Chem. B, 2017, 5, 3862-3869.

[36] Reja, S. I.; Khan, I. A.; Bhalla, V.; Kumar, M., A TICT based NIR-fluorescent probe for human serum albumin: a pre-clinical diagnosis in blood serum. Chem. Commun., 2016, 52, 1182-1185.

[37] Hansch, C.; Leo, A.; Taft, R. W., A Survey of Hammett Substituent Constants and Resonance and Field Parameters. Chem. Rev., 1991, 91, 165-195.

[38] Chen, W.; Xu, S.; Day, J. J.; Wang, D.; Xian, M., A General Strategy for Development of Near-Infrared Fluorescent Probes for Bioimaging. Angew. Chem. Int. Ed., 2017, 56, 16611-16615.

[39] Hu, R.; Lager, E.; Aguilar-Aguilar, A.; Liu, J.; Lam, J. W. Y.; Sung, H. H. Y.; Williams, I. D.; Zhong, Y.; Wong, K. S.; Peña-Cabrera, E.; Tang, B. Z., Twisted 
Intramolecular Charge Transfer and Aggregation-Induced Emission of BODIPY Derivatives. J. Phys. Chem. C., 2009, 113, 15845-15853;

[40] Gao, S.; Wei, G.; Zhang, S.; Zheng, B.; Xu, J.; Chen, G.; Li, M.; Song, S.; Fu, W.; Xiao, Z.; Lu, W., Albumin tailoring fluorescence and photothermal conversion effect of near-infrared-II fluorophore with aggregation-induced emission characteristics. Nat. Commun., 2019, 10, 2206-2220.

[41] Xiong, J.; Wang, K.; Yao, Z.; Zou, B.; Xu, J.; Bu, X.-H., Multi-Stimuli-Responsive Fluorescence Switching from a Pyridine-Functionalized Tetraphenylethene AIEgen. ACS Appl. Mater. Inter., 2018, 10, 5819-5827.

[42] Luo, J.; Xie, Z.; Lam, J. W.; Cheng, L.; Chen, H.; Qiu, C.; Kwok, H. S.; Zhan, X.; Liu, Y.; Zhu, D.; Tang, B. Z., Aggregation-induced emission of 1-methyl-1,2,3,4,5pentaphenylsilole. Chem. Commun., 2001, 18, 1740-1741.

[43] Huang, H.; Zhou, Y,; Wang, Y.; Cao, X.; Han, C.; Liu, G.; Xu, Z.; Zhan, C.; Hua, H.; Peng, Y.; Yan, P.; Cao. D., Precise Molecular Design for B-N Modified Polycyclic Aromatic Hydrocarbon Toward Mechanochromic Material. J. Mater. Chem. A., 2020, 8, 22023-22031.

[44] Yang, W.; Liu, C.; Lu, S.; Du, J.; Gao, Q.; Zhang, R.; Liu, Y.; Yang, C., AIEactive smart cyanostyrene luminogens: polymorphism-dependent multicolor mechanochromism. J. Mater. Chem. C., 2018, 6, 290-298. 
[45] Ma, Z.; Ji, Y.; Lan, Y.; Kuang, G.; Jia, X., Two novel rhodamine-based molecules with different mechanochromic and photochromic properties in solid state. J. Mater. Chem. C., 2018, 6, 2270-2274.

[46] Li, G.; Xu, Y.; Zhuang, W.; Wang, Y., Preparation of organic mechanochromic fluorophores with simple structures and promising mechanochromic luminescence properties. $R S C A d v .$, 2016, 6, 84787-84793.

[47] Morris, G. M.; Huey, R.; Lindstrom, W.; Sanner, M. F.; Belew, R. K.; Goodsell, D. S.; Olson, A. J., AutoDock4 and AutoDockTools4: Automated docking with selective receptor flexibility. J. Comput. Chem., 2009, 30, 2785-2791.

[48] Orlando, B. J.; Malkowski, M. G., Crystal structure of rofecoxib bound to human cyclooxygenase-2. Acta Crystallogr. F-Struct. Biol. Commun., 2016, 72, 772-776.

[49] Yu, H.-P.; Shi, L.-Y.; Lu, W.-H.; Su, Y.-H.; Li, Y.-Y.; Xu, S.-Q., Expression of cyclooxygenase-2 (COX-2) in human esophageal cancer and in vitro inhibition by a specific COX-2 inhibitor, NS-398. J Gastroen Hepatol., 2004, 19 (6), 638-642.

[50] Zhang, Y.; Dong, S.; Xu, R.; Yang, Y.; Zheng, Z.; Wang, X.; Ren, R.; Sun, R.; Li, M.; Yang, H.; Huang, Y.; Zhou, F.; Zheng, A., Prognostic and predictive role of COX2, XRCC1 and RASSF1 expression in patients with esophageal squamous cell carcinoma receiving radiotherapy. Oncol Lett., 2017, 13 (4), 2549-2556.

[51] Moon, H.; White, A. C.; Borowsky, A. D., New insights into the functions of Cox2 in skin and esophageal malignancies. Exp. Mol. Med., 2020, 52 (4), 538-547. 
Table of Contents Graphic

Table of Contents (TOC)

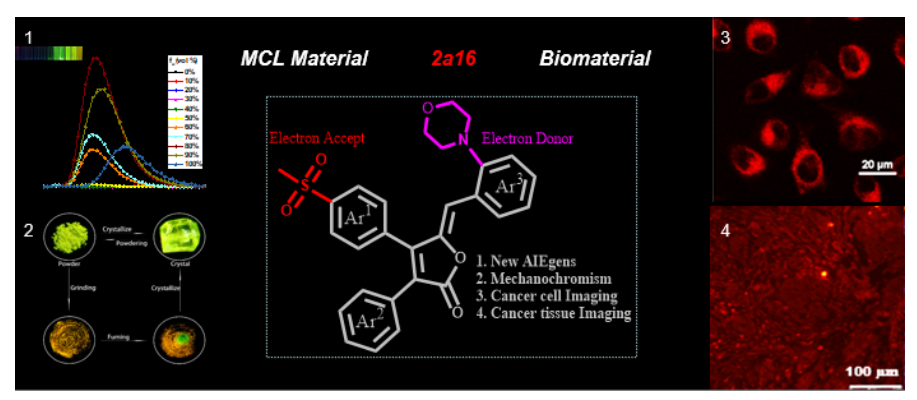

